\title{
BMJ Open Transvaginal natural orifice transluminal endoscopic surgery (vNOTES) adnexectomy for benign pathology compared with laparoscopic excision (NOTABLE): a protocol for a randomised controlled trial
}

Jan Filip Baekelandt, ${ }^{1}$ Peter A De Mulder, ${ }^{2}$ Ilse Le Roy, ${ }^{2}$ Chantal Mathieu, ${ }^{3,4}$ Annouschka Laenen, ${ }^{5}$ Paul Enzlin, ${ }^{6}$ Steven Weyers, ${ }^{7}$ Ben W J Mol, ${ }^{8,9}$ Jan J A Bosteels S $^{12,7,10}$

To cite: Baekelandt JF, De Mulder PA, Le Roy I, et al. Transvaginal natural orifice transluminal endoscopic surgery (vNOTES) adnexectomy for benign pathology compared with laparoscopic excision (NOTABLE): a protocol for a randomised controlled trial. BMJ Open 2018;8:e018059. doi:10.1136/ bmjopen-2017-018059

- Prepublication history and additional material for this paper are available online. To view these files, please visit the journal online (http://dx.doi. org/10.1136/bmjopen-2017018059).

Received 2 June 2017

Revised 14 November 2017 Accepted 24 November 2017

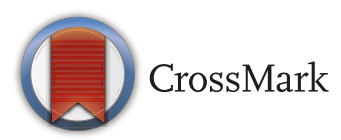

For numbered affiliations see end of article.

Correspondence to Dr Jan Filip Baekelandt; jan.baekelandt@imelda.be

\section{ABSTRACT}

Introduction Natural orifice transluminal endoscopic surgery (NOTES) uses natural orifices to access the cavities of the human body to perform surgical interventions. NOTES limits the magnitude of surgical trauma and potentially reduces postoperative pain. Our group published a protocol on a randomised study comparing transvaginal NOTES (VNOTES) versus laparoscopy for hysterectomy (HALON). We simultaneously designed a similar randomised controlled trial (RCT) comparing vNOTES with laparoscopy for adnexectomy. To the best of our knowledge, this is the first RCT comparing VNOTES with laparoscopy for adnexal surgery.

Methods and analysis The methodology of the Notes Adnexectomy for Benign Indication versus Laparoscopic Excision (NOTABLE) study is similar to that of the HALON trial. Women aged 18-70 years with an indication for benign adnexal surgery will be eligible. We will use stratification according to adnexal size. Entrants will be randomised to the laparoscopic treatment (control) or VNOTES (intervention). Participants will be evaluated on days $0-7$ and at 3 and 6 months. The primary outcome will be the proportion of women successfully treated by removing an adnexa by the allocated technique without conversion. We will collect the following data (secondary outcomes): proportion of women hospitalised on the day of surgery, postoperative pain scores measured two times per day from day 1 to 7 , total dosage of pain killers used from day 1 to 7 , hospital readmission during the first 6 weeks, dyspareunia and sexual well-being at baseline, 3 and 6 months using a validated questionnaire (Short Sexual Functioning Scale), health-related quality of life at baseline, 3 and 6 months after surgery using a validated questionnaire (EQ-5D-3L), duration of surgical intervention, infection or other surgical complications and direct costs up to 6 weeks following surgery. For the primary outcome measure, a one-sided $95 \% \mathrm{Cl}$ of the difference in the proportions of women with a successful removal of the uterus by the randomised technique will be estimated. Noninferiority will be concluded when $15 \%$ lies above the upper limit of this $95 \% \mathrm{Cl}$.

\section{Strengths and limitations of this study}

- This study is a randomised controlled trial.

- The patients, the outcome assessors and the personnel are blinded in this trial.

- This is a single-centre study.

- The generalisability of this study to a 'real-life' setting is limited due to the experimental setting of the study.

- The use of non-therapeutic incisions for blinding may confound the outcome pain.

Ethics and dissemination The study was approved on 1 December 2015 by the EthicsCommitteeof the Imelda Hospital (registration no: 689), Bonheiden, Belgium. We aim to present the final results of the NOTABLE trial in peer-reviewed journals and at scientific meetings within 4 years after the start of the recruitment.

Trial registration number NCT02630329.

\section{BACKGROUND}

Laparoscopic surgery has reduced surgical morbidity and mortality. 'Minimally invasive surgery' has moved even further forward with newer techniques such as single incision laparoscopic surgery and natural orifice transluminal endoscopy (NOTES) with or without robot assistance.

The NOTES technique uses any natural orifice (mouth, vagina, urethra or rectum) as a possible access route facilitating a surgical intervention in a cavity of the human body. Clinical researchers at Johns Hopkins University first reported its use in 2004 in a preclinical trial using an animal model. ${ }^{1}$ Ever since the clinical application of NOTES has been 
reported in many surgical procedures in ways that seem to defy human imagination, appendectomy and cholecystectomy have been performed using the mouth and the stomach as the access route. ${ }^{23}$ The technique seems feasible and safe in the hands of experienced surgeons beyond their surgical learning curve. Observational evidence (mostly case reports) has reported, moreover, that NOTES may cause less postoperative pain, a shorter length of hospital stay, less complications and last but not least, for female patients, improved cosmetic results. The feasibility of scar-free surgery in combination with reduced wound (trocar) complications may be tempting for patients and their care providers. This may be a strong facilitator for the widespread implementation of this new surgical approach.

NOTES has gained popularity among general surgeons, urologists and gastroenterologists over the past few years, and its feasibility and safety in these domains have been reported. ${ }^{4}$ Although NOTES may be performed using various entries including the stomach, oesophagus, bladder and rectum, the majority of NOTES procedures in women have been performed through the vagina. ${ }^{5}$ This is not surprising because the colpotomy technique has been used widely in vaginal prolapse surgery and for benign adnexal surgery involving the extraction of large specimens. Its use has been reported as a safe access. ${ }^{67}$ Two variants of NOTES have been described in the present literature. Hybrid NOTES combines the access through the vagina with transabdominal assistance; pure NOTES refers to procedures that involve only transluminal access.

The removal of one or both adnexa using a transvaginal NOTES (vNOTES) approach was described for the first time by Lee and coworkers in 2012. ${ }^{8}$ vNOTES adnexectomy for benign pathology was introduced at our department by the first author (JFB) in 2013. Our group published three small case series on adnexal removal $(\mathrm{n}=20),{ }^{9}$ salpingectomy $(\mathrm{n}=5)^{10}$ and hysterectomy $(\mathrm{n}=10)^{11}$ by vNOTES during the period between November 2013 and February 2015. We also published the protocol of the laparoscopy for hysterectomy (HALON) study randomly comparing NOTES and laparoscopy for doing hysterectomy in women with benign gynaecological disease. ${ }^{12}$ The recruitment of the HALON study was finished recently (24 February 2017). The final data analysis of the HALON study is foreseen for September 2017.

\section{Objectives and hypotheses}

We started our research by doing a systematic review (SR) of the literature. We searched MEDLINE, EMBASE and the Cochrane Library from inception to 1 August 2015 using a combination of MeSH terms and key words for 'colpotomy' and 'adnexal diseases' or 'adnexal mass'. We aim to publish the results of this systematic search of the literature and a critical appraisal of the retrieved evidence in a separate SR: we will adhere to the Preferred Reporting Items for Systematic Review and Meta-Analysis Protocols guidelines ${ }^{13}$ for the protocol of this SR. The protocol of the SR has been registered in the International Prospective
Register of Systematic Reviews (PROSPERO) at the Centre for Reviews and Dissemination (CRD), University of York, UK ${ }^{14}$ as CRD42016033670. To the best of our knowledge, no randomised controlled studies comparing vNOTES with the transabdominal laparoscopic approach for removal of one or both adnexa have been published in the literature. The main objective of the NOTABLE study is to study the effectiveness of vNOTES for successfully removing one or both adnexa for benign gynaecological disease using the classical laparoscopic approach as the established effective technique. The rationale and the objectives of NOTABLE are in accordance with the principles outlined by the Idea Development Exploration Assessment Long term study (IDEAL) collaboration. ${ }^{15-17}$

Our primary study hypothesis is that vNOTES is not inferior to transabdominal laparoscopy for removing one or both adnexa for a benign gynaecological indication without having to convert to another technique. vNOTES may offer several advantages including the avoidance of abdominal scars, less need for hospital admission and possibly less postoperative pain.

\section{METHODS}

\section{Trial design and study analysis}

The NOTABLE study should be considered as a pilot study. It is a single-centre parallel-group double-blinded (patient and outcome assessor) randomised trial conducted at the Department of Gynaecology of the Imelda Hospital in Bonheiden. This is a general hospital in Belgium serving an estimated population of 150000 people. All women aged 18-70 years bound to undergo removal of one or both adnexa for benign gynaecological disease will be informed about the NOTABLE study, and they will be invited to participate in the study, if eligible. The baseline characteristics of eligible women not wishing to give informed consent for participating in the study will be recorded as well as the reason for declining to participate. All surgical procedures (vNOTES and laparoscopy) will be done by one surgeon (JFB) who is equally skilled in both techniques. The surgeon has been using the vNOTES approach for various interventions (salpingectomy for Extra-Uterine Gravidity (EUG), adnexectomy and hysterectomy) since November 2013. JFB is also the surgeon performing the hysterectomies in the HALON trial. The surgeon cannot be blinded, but the allocated treatment will be concealed. We will use a non-inferiority study design to test the effectiveness of vNOTES compared with laparoscopy. The protocol adheres to the Standard Protocol Items: Recommendations for Interventional Trials standards (http://www.spirit-statement. $\mathrm{org} /$ ). The study protocol of the NOTABLE trial is very similar to that of the earlier published HALON study. ${ }^{12}$

\section{Study dates}

The first patient was included on 15 January 2016. On 22 May 2017, 38 of the targeted 70 participants were 
recruited. Anticipated date of study completion is estimated in May 2018.

\section{Participants}

NOTABLE will recruit eligible women aged 18-70 years, regardless of parity, who need the removal of one or both adnexa for a benign adnexal disease and who provide informed consent prior to surgery.

Exclusion criteria are as follows:

- history of rectal surgery

- suspected rectovaginal endometriosis

- suspected malignancy

- history of pelvic inflammatory disease

- active lower genital tract infection

- virginity

- pregnancy

- failure to provide written informed consent.

\section{Intervention, procedures and standard care}

On the day of the surgery, all patients are admitted to the day care unit. A nurse administers clindamycin vaginal cream on admission.

Under general anaesthesia, the patient is positioned in a vacuum mattress in the classical lithotomy. An alcoholic Betadine solution is used for disinfection of the vagina, vulva and abdomen before draping. A Foley catheter is inserted into the bladder. In accordance with hospital protocol, the anaesthesiologist will administer cefazolin $2 \mathrm{~g}$ and metronidazole $1.5 \mathrm{~g}$ intravenous prior to incision for prophylaxis against infection to all women of both treatment arms. In both groups, a $30^{\circ}$ rigid endoscope is used.

\section{Control group: laparoscopic technique}

The surgeon will start the procedure by making a small vertical intraumbilical skin incision. A Veress needle is introduced into the peritoneal cavity; the tip position is checked with a Semm test before insufflating carbon dioxide $\left(\mathrm{CO}_{2}\right)$ until a maximal intraperitoneal pressure of $15 \mathrm{~mm} \mathrm{Hg}$. A $10 \mathrm{~mm}$ trocar is inserted through the umbilicus after removal of the Veress needle. An optic is inserted to inspect the peritoneal cavity. The operating table is tilted in the Trendelenburg position. Two $5 \mathrm{~mm}$ trocars are placed under direct vision in the suprapubic region and in the left iliac fossa lateral of the epigastric vessels. The small intestine is lifted out of the pelvis.

The ureter is identified, but no retroperitoneal dissection is performed unless indicated. The proximal end of the Fallopian tube is coagulated at its origin in the uterus using a reusable bipolar grasping forceps before being cut with cold microscissors. The ovarian and infundibulopelvic ligament are coagulated and cut. After resection, the adnexa is placed in an endobag (Memobag; Teleflex, Wayne, Pennsylvania, USA). When indicated, the same procedure is repeated for the contralateral side.

After confirmation of haemostasis, the peritoneal cavity is rinsed. No drains are left in the peritoneal cavity unless necessary, for example, problematic haemostasis. The
$5 \mathrm{~mm}$ trocars are be removed under direct vision. The purse string of the endobag is pulled through the $10 \mathrm{~mm}$ trocar on removal of the optic. The umbilical incision is extended vertically in caudal direction, but not more than $2.5 \mathrm{~cm}$. The fascia and peritoneum are opened, and the proximal end of the endobag is pulled through the incision without causing any rupture if possible. If not possible, the endobag will be opened, and the content of the cyst will be aspirated to reduce the volume of the adnexa. The aspirated fluid will be sent for cytological evaluation. The endobag will then be removed with the adnexa inside it.

The fascia is closed using a Vicryl-1 running suture. The umbilicus and other incisions are disinfected with Betadine solution. All skin incisions are closed with a Monocryl $3 / 0$ intradermal suture and approximated using steristrips. The wound sites are covered with a wound dressing. A vaginal plug (Betadine gauze $10 \mathrm{~cm} \times 5 \mathrm{~m}$ ) is inserted into the vagina. After 3 hours, the Foley catheter and the vaginal plug are removed.

\section{Intervention group: vNOTES}

The surgeon makes three non-therapeutic superficial skin incisions on exactly the same location as in the classical laparoscopic approach in all women allocated to the vNOTES group to blind study participants and the outcome assessor to the allocated technique. A $2.5 \mathrm{~cm}$ posterior colpotomy is made using a cold knife. The pouch of Douglas is opened using scissors. A Gelpoint Mini (Applied Medical), used as vNOTES port, is inserted into the pouch of Douglas. $\mathrm{CO}_{2}$ is insufflated until a maximal intraperitoneal pressure of $15 \mathrm{~mm} \mathrm{Hg}$. An optic is inserted to inspect the peritoneal cavity. The operating table is tilted in the Trendelenburg position. The small intestine is lifted out of the pelvis.

The ureter is identified, but no retroperitoneal dissection is performed unless indicated. The proximal end of the Fallopian tube is coagulated at its origin into the uterus using a reusable bipolar grasping forceps and cut using microscissors. The ovarian and infundibulopelvic ligament are coagulated and cut. The adnexa is removed. When indicated, the procedure is repeated for the contralateral side. After confirmation of haemostasis, the peritoneal cavity is rinsed.

Small benign-looking adnexa is removed directly through the wound protector part of the vNOTES port. Large adnexa or adnexa that appears macroscopically suspicious is placed in an endobag (Memobag; Teleflex). The purse string of the endobag is pulled through the wound protector, and the purse string released. The content of the cyst is aspirated to reduce the volume of the adnexa. The endobag is then removed with the adnexa inside it. The vNOTES port is removed.

The colpotomy is closed using three interrupted figure-of-eight Vicryl 2/0 sutures. A vaginal plug (Betadine gauze $10 \mathrm{~cm} \times 5 \mathrm{~m}$ ) is inserted into the vagina. After 3 hours, the Foley catheter and the vaginal plug are removed. 
In the majority of patients, it is feasible to perform a successful vNOTES or laparoscopic adnexectomy. Women in whom the intended approach has to be abandoned for an alternative intervention will not be excluded or withdrawn from the NOTABLE trial but will be followed up further. It is anticipated that most included patients with a normal cancer antigen 125 value, and benign features of the ovary on ultrasound will not require other interventions besides the removal of the adnexa. If the responsible clinician judges that additional treatment is necessary at the time of the surgery or afterwards, this will be recorded and the patient will not be withdrawn from the study. However, if there is a preoperative indication for additional surgery during the same procedure, these patients will be excluded from recruitment to the NOTABLE trial.

The anaesthesiologists involved in the clinical trial have developed a standardised protocol to insure that the pain management is identical for both groups. The outcome assessor (JJAB) and the patient are both blinded to the surgical approach used. The patient makes the decision to be discharged from the day care unit on the evening of the procedure or to be admitted to an in-hospital nursing ward for the night. The outcome assessor can only overrule the patient's decision in the interest of her health, for example, when surgical complications were recorded in the surgical report or when vital parameters indicate a life-threatening condition. Before discharge, all patients are given a standard list of instructions to avoid physical work, exercise and sexual intercourse for 4 weeks after the intervention.

All participants, regardless of being at home or in hospital, are requested to use a Visual Analogue Scale (VAS) two times per day to measure postoperative pain from day 1 to day 7 following surgery. Adequate instructions on how to use the VAS measuring tool are given on an individual basis by a dedicated nurse of the day care unit. One measurement is made in the evening before going to bed after physical activity (active), and another is made in the morning after bed rest at night (rest). All patients are asked to note the name, dosage and route of administration of any analgesic drug taken from day 1 to 7 in a pain log book.

\section{Outcome measure}

We searched the COMET $^{18}$ database for a core outcome set for adnexectomy (general settings) in gynaecology (health area-disease category) in women (target population: sex) aged 18 to 70 years (target population: age): no core outcome set relevant to laparoscopic removal of adnexa was identified. ${ }^{19}$

\section{Primary outcome measure}

The proportion of women successfully treated by removing one or both adnexa without spill by the allocated technique as randomised will be measured as the primary outcome of effectiveness.
Secondary outcome measures

The secondary outcomes are as follows:

- The proportion of women hospitalised on the day of surgery based on their own preference.

- Postoperative pain scores measured using VAS $^{20}$ two times per day from day 1 to 7 .

- The total dosage of pain killers taken during the first week following surgery.

- Postoperative infection defined by lower abdominal pain with fever $>38^{\circ} \mathrm{C}$ and positive clinical signs or laboratory findings detected during the first 6 weeks of surgery.

- Intraoperative or postoperative complications classified according to the Clavien-Dindo classification ${ }^{21}$ detected during the first 6 weeks of surgery.

- Readmission to hospital during the first 6 weeks of surgery.

- Occurrence and severity of pain on sexual intercourse self-reported by the study participants at baseline, 3 and 6 months by using a simple questionnaire and VAS.

- Sexual well-being at baseline, 3 and 6 months by self-reporting the Short Sexual Functioning Scale (SSFS). ${ }^{22}$

- Health-related quality of life (QoL) at baseline, 3 and 6 months after surgery by self-reporting using a validated questionnaire (EQ-5D-3L).

- The duration of the surgical intervention measured in minutes from the insertion of the bladder catheter to the end of vaginal/abdominal wound closure.

- Direct costs for both techniques up to 6 weeks following surgery.

The SSFS and the EQ-5D-3L questionnaires were validated in Dutch and presented to the participants in their mother tongue.

\section{Randomisation and blinding}

Participants will be randomly allocated to one of both treatment arms (vNOTES vs laparoscopy). We will use a computer-generated randomisation schedule generated by the management assistant of our department. We will use a stratification into three categories (A, B or $\mathrm{C}$ ) according to the size of the cyst on transvaginal ultrasound (0 to $5 \mathrm{~cm}, 5$ to $10 \mathrm{~cm}$ and larger than $10 \mathrm{~cm}$ ). Sequentially numbered, opaque, sealed envelopes will be used to ensure allocation concealment for the surgeon and the outcome assessor. The management assistant will safeguard the allocation code until the last visit of the last patient. The management assistant will not be involved in the outcome assessment or the data collection.

All participating women and the outcome assessor will be blinded to the allocation by the use of non-therapeutic skin incisions. It is impossible to blind the surgeon. In case of life-threatening adverse events, the outcome assessor will notify the surgeon to enable further treatment without the need for non-blinding the patient. The use of the vNOTES technique avoids the use of abdominal incisions. Participants allocated to the vNOTES arm will have 
three superficial non-therapeutic skin incisions similar to those routinely done with the laparoscopic technique. This enables blinding all study participants, personnel and the outcome assessor. The wound dressings of all women will be left untouched until the postoperative visit on day 7 . The practice of using non-therapeutic skin incisions has been reported in some surgical trials to minimise performance and detection bias when measuring subjective outcomes (eg, pain) ${ }^{23}$ The decision to use non-therapeutic skin incisions is justified by the risk to benefit ratio of the two interventions under comparison. ${ }^{24}$ Its use in the HALON and NOTABLE trials has been intensively discussed among the investigators. The written approval with the Belgian unique study identifier B689201526268 was sent to the Federal Agency for Medicines and Health Products (FAMHP) in Brussels.

\section{Statistical methods}

Sample size calculation

A sample size calculation was done for the primary outcome only. An appropriate level of statistical power was applied to preclude any clinically important inferiority of vNOTES compared with laparoscopy. The assumptions for the sample size calculation are based on evidence retrieved from two sources: a randomised study comparing the excision of mature dermoid cysts using colpotomy with laparoscopic assistance versus colpotomy without laparoscopy ${ }^{25}$ and an SR with meta-analysis comparing single port laparoscopy versus conventional laparoscopy in benign adnexal disease. ${ }^{26}$ An important consideration in any adnexal mass surgery is the inadvertent opening of the ovarian capsule of an unsuspected malignancy resulting in the spill of malignant cells into the abdominal cavity. Based on a $2.4 \%$ failure rate to remove dermoid cysts by colpotomy using laparoscopic assistance ${ }^{25}$ and a $0 \%$ conversion rate from laparoscopy to laparotomy, ${ }^{26}$ we assumed that the successful removal of adnexal cysts without spill would be feasible in $95 \%$ of all cases. The sample size was calculated with a one-sided test for non-inferiority for the primary outcome. The vNOTES approach may be the treatment of choice for women because it avoids scars. We assume that vNOTES would be the preferred technique even when $15 \%$ less women had in the end a successful removal of a benign adnexal mass by using vNOTES compared with laparoscopy with its unavoidable scars. Non-inferiority will be concluded when $15 \%$ lies above the upper limit of the 95\% CI calculated for the difference in the proportion of women successfully treated with either technique. To achieve $80 \%$ power to demonstrate non-inferiority under the assumption of similar success rates of $95 \%$ in both groups, a sample size of 54 participants (27 women per group) will be required. We increased the target sample size to 64 participants (32 women per group) to account for a dropout rate of $15 \%$. Based on the power calculations for the primary outcome, the use of three strata for the randomisation and assuming a loss-to-follow-up rate of $15 \%$, we decided to include 66 study participants in the NOTABLE trial.

\section{Statistical analyses}

\section{General principles}

For all baseline and outcome variables, the number of available measurements and the number of missing values will be given. A probability $(\mathrm{P})$ less than 0.05 will be considered to be significant. Analysis will be performed by intention-to-treat, as recommended in the Consolidated Standards of Reporting Trials (CONSORT) statement. ${ }^{27}$ Since the study compares two regular interventions and is expected to recruit during a reasonably limited period, interim analyses will not be performed.

Categorical data will be reported as absolute numbers and percentages. Normally distributed continuous variables will be summarised as means with SDs, and non-normally distributed continuous variables will be reported as medians with IQR. Main analyses will not impute missing values.

All analyses will be performed using SAS software (V.9.4 of the SAS System for Windows).

\section{DESCRIPTIVE ANALYSES}

Study population-baseline characteristics

- Mean age $( \pm \mathrm{SD})$,

- Mean body mass index (BMI) $( \pm \mathrm{SD})$,

- Mean number of natural vaginal births $( \pm \mathrm{SD})$,

- Mean number of abdominal/pelvic surgical interventions $( \pm \mathrm{SD})$,

- Mean weight of the uterus $( \pm \mathrm{SD})$.

\section{STUDY ENDPOINTS}

\section{Main study parameter/endpoint}

Differences in the proportions of women successfully treated by removing the uterus by the intended technique without conversion to another approach.

\section{Secondary study parameters/endpoints}

- Proportions of women hospitalised on the day of surgery;

- Postoperative pain scores, measured using VAS two times per day from day 1 to 7 self-reported by the study participants;

- Total dose analgesics used during the first week following surgery;

- Incidence of postoperative infection during the first 6 weeks of surgery;

- Incidence of intraoperative complications;

- Incidence of postoperative complications during the first 6 weeks following surgery;

- Incidence of readmission during the first 6 weeks of surgery;

- Incidence of dyspareunia recorded by the participants at baseline, 3 and 6 months by self-reporting using a simple questionnaire;

- Severity of dyspareunia recorded by the participants at baseline, 3 and 6 months by self-reporting using VAS; 
- Sexual well-being at baseline, 3 and 6 months by self-reporting the SSFS QoL at baseline, 3 and 6 months by self-reporting the EQ-5D-3L questionnaire;

- Duration of surgery;

- Total costs of both intervention surgeries.

\section{STATISTICAL ANALYSIS}

For the primary outcome measure, a one-sided $95 \%$ CI of the difference in the proportions of women with a successful removal of the uterus by the intended technique as randomised will be estimated. Non-inferiority will be concluded when $15 \%$ lies above the upper limit of this $95 \%$ CI.

For the manuscript, all above listed secondary outcomes will be compared between the two groups. These data will be reported as vNOTES versus laparoscopy.

For dichotomous secondary outcome measures, comparisons between the two arms will be performed by applying Fisher's exact test or $\mathrm{X}^{2}$ test, as appropriate.

Cross-sectionally measured continuous secondary outcomes will be analysed using an independent t-test or Mann-Whitney U test, as appropriate.

Longitudinally measured continuous secondary outcomes will be analysed using multilevel modelling. Differences in evolution between both treatment groups will be compared by means of a time by group interaction. In absence of such an interaction, mean differences will be compared over all time points. Outcome scores will be transformed if required to meet model assumptions.

All statistical analyses will be done by an experienced biostatistician (AL) who is a coinvestigator. After data cleaning, the management secretary will send the non-blinded data to the biostatistician after the last visit of the last patient. The biostatistician will do all the analyses without any assistance of the other investigators who will remain blinded until all data have been analysed.

The following strategy will be used in case of missing data. In case of a single item response missing, the data will be imputed from given values. In cases where more than one item is missing or an entire form is missing, imputation will not be attempted. We will assess whether the obtained results are robust to the methods used to handle missing data, by performing a sensitivity analysis.

\section{MONITORING}

NOTABLE is a small trial; therefore, a data monitoring committee is not needed.

All adverse events reported spontaneously by the participant or observed by the investigator or his staff will be recorded. Infection and perioperative or postoperative complications will be assessed as secondary outcomes until 6 weeks after surgery. We will inform the family physician of all participants in order to assess all possible unintended effects of the trial intervention and promote to report all possible adverse events anonymously using the participant's unique study number to an email address ( NOTES@imelda.be).We will use descriptive statistics for data analysis although the trial is not adequately powered to detect important differences in rates of uncommon adverse events. Given the limited resources and the singlecentre design, there will be no auditing of the conduct of the trial. We will review patient enrolment, consent and eligibility on a regular basis to promote data quality and to preserve trial integrity. The distribution of the allocation to the study groups will be blindly checked by the study secretary at $30 \%, 60 \%$ and $90 \%$ of the recruitment and discussed with the study statistician and the principal investigators.

\section{RESULTS}

\section{Participant flow diagram}

Figure 1 shows the study flow reported as outlined by the CONSORT (figure 1).

\section{Recruitment time frame}

All potentially eligible women aged 18 to 70 years, regardless of parity, in need of adnexal surgery for benign gynaecological disease without exclusion criteria will be invited to participate in the trial. Only eligible women with written informed consent obtained before randomisation will be finally included in the NOTABLE trial.

We perform 36 interventions for adnexal surgery by laparoscopy for benign gynaecological disease at our Department of Gynaecology per year. The recruitment period of NOTABLE to meet the sample size will be approximately 2 years. Including the follow-up period of 6 months after the last patient last visit and the time required to perform data analysis and reporting ( 6 months to 1 year), we estimate that the total study period will be at least 3 years.

\section{Data collection}

We will record the following patient characteristics at baseline: age, BMI, the number of vaginal births, previous abdominal or pelvic surgery (C sections included), adnexal size, concomitant medication, dyspareunia questionnaire and the SSFS.

On the day of surgical intervention (day 0 ), we will record the following data: the duration of the surgical intervention, the successful removal of the adnexa by the technique as allocated without conversion to another technique with or without spilling (into the peritoneal cavity or the endobag), hospitalisation of the participant on the day of the surgical intervention based on her own preference, the total dosage of analgesics used at the recovery and day care unit and the maximum VAS pain score on the day 0 .

After 1 week at visit day 7 , the outcome assessor will collect the pain scores as self-reported by the study participants two times per day from day 1 to day 7 using VAS. The outcome assessor will also collect data on the total dosage of pain killers used during the first postoperative week.

At visit day 7 and day 42, the outcome assessor will record the following data: pelvic infection defined by 


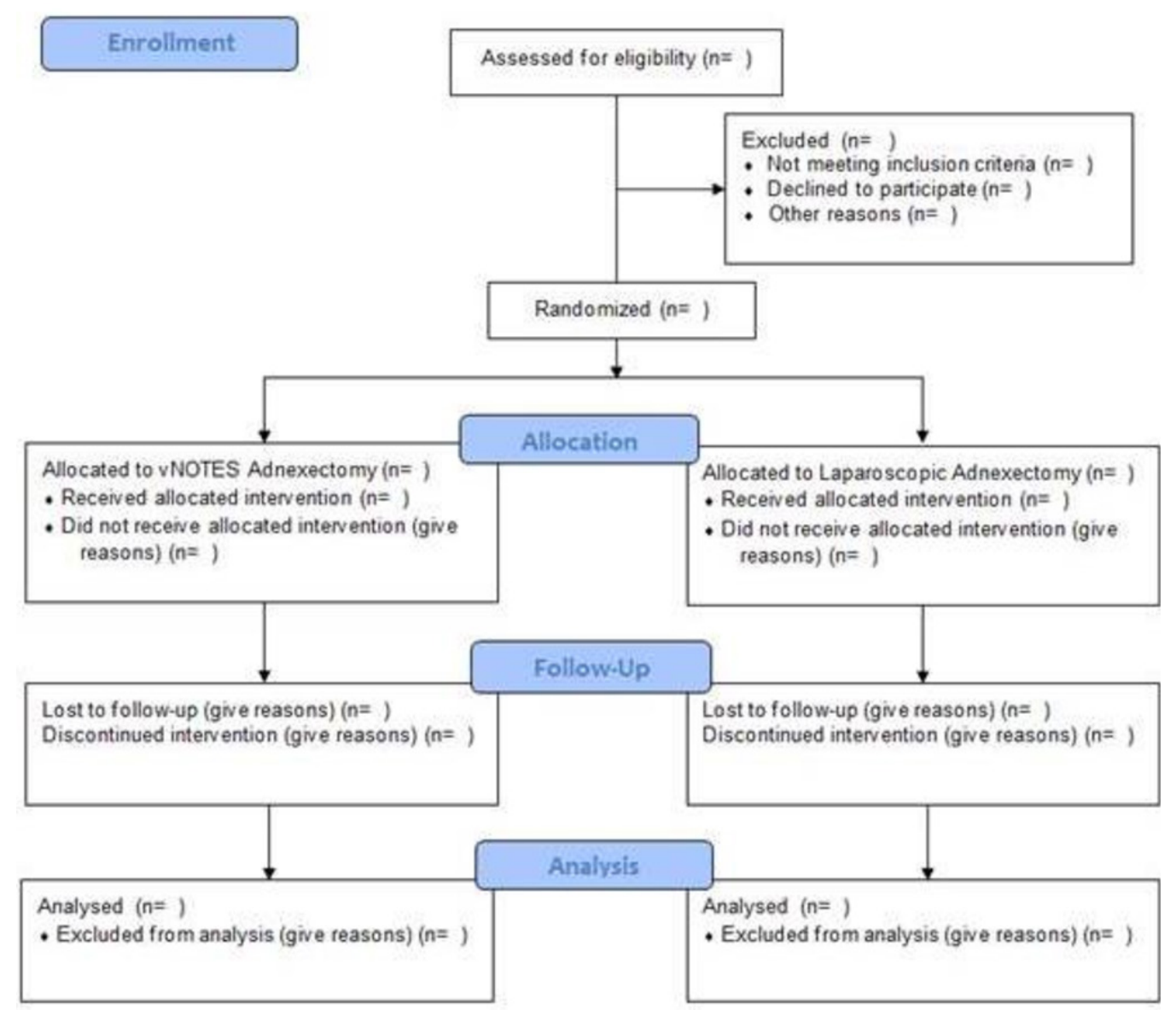

Figure 1 CONSORT (Consolidated Standards of Reporting Trials) 2010 flow diagram. vNOTES, transvaginal natural orifice transluminal endoscopic surgery.

lower abdominal pain with fever $>38^{\circ} \mathrm{C}$ and positive clinical signs or laboratory findings, readmission to hospital and the occurrence of other postoperative complications classified according to the Clavien-Dindo classification.

On months 3 and 6 following surgery, the dyspareunia questionnaires, the EQ-5D-3L and the SSFS questionnaires will be filled in by the study participants and collected by regular mail. The management assistant will oversee this process and send reminders until all questionnaires have been received. We refer to table 1 for an overview of the data collection process (table 1).

\section{DISCUSSION}

\section{Interpretation, limitations and generalisability}

The NOTABLE trial is a randomised pilot study on the efficacy of the vNOTES technique. All surgical procedures in the NOTABLE study are done by one single surgeon (JFB) who is equally skilled in using both techniques under comparison. The surgeon has been using the vNOTES approach since November 2013. During this 2-year period, the new technique and suitable instruments used were pilot-tested and subsequently fine-tuned by the usual 'trial and error' method used for centuries in surgical practice. ${ }^{17}$ The feasibility and preliminary safety of the new technique were reported in three observational studies performed at our department ${ }^{9-11}$ in accordance with the principles outlined in the three article series on the IDEAL statement. ${ }^{15-17}$ According to the terminology used by the IDEAL collaboration, ${ }^{17}$ this study should be classified as an IDEAL stage $2 \mathrm{~b}$ trial. The full Patient Intervention Control Outcome (PICO) research question is as follows: will a surgeon who is equally skilled at performing both techniques, and beyond his learning curve for the new technique (vNOTES), succeed in removing one or both adnexa in women with benign gynaecological disease at least as often with the new pilot-tested vNOTES approach compared with the standard transabdominal laparoscopic approach without having to convert to any other technique. An intraoperative decision to remove an adnexa via laparoscopy and not via vNOTES due to, for example, a large specimen or an atrophic vagina may better be defined as a pre-emptive conversion, as it has less clinical implications than a conversion from laparoscopy to laparotomy. However, for the sake of unambiguity in this trial, we decided to count as a conversion every case that was not treated by the allocated technique, whether the conversion was pre-emptive or not.

NOTABLE aims to measure efficacy of vNOTES for removing one or both adnexa (Can vNOTES work under ideal experimental conditions?). The NOTABLE trial 
Table 1 Patient's characteristics and data collection

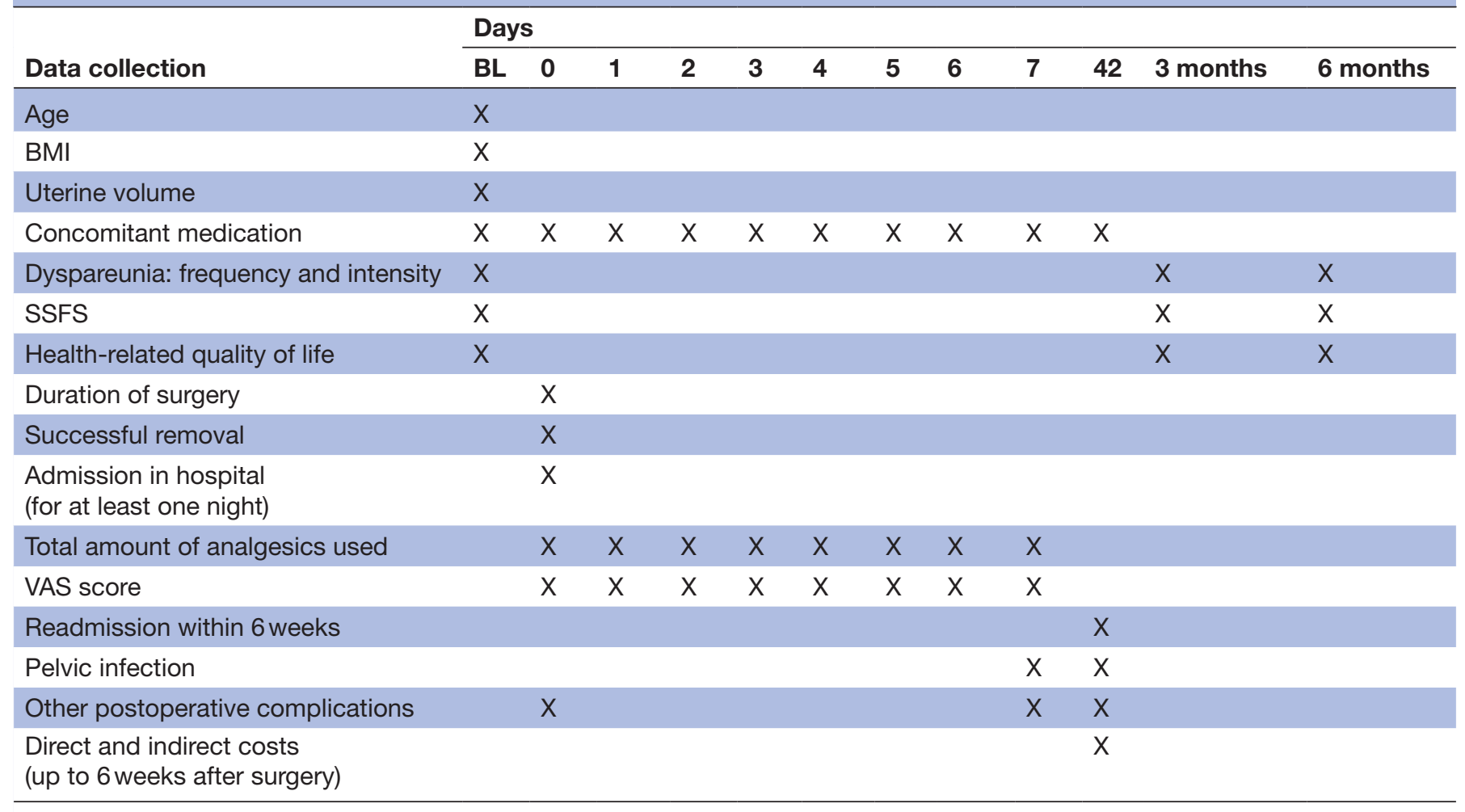

BL, baseline; BMI, body mass index; SSFS, Short Sexual Functioning Scale; VAS, Visual Analogue Scale.

does not address the effectiveness of the new intervention at this moment (Does vNOTES work in a real-life setting?). The conditions in NOTABLE are truly experimental and in many instances opposed to 'real life' practice: all women are always treated by the most experienced surgeon equally skilled in using both techniques, all women receive more attention during this trial than the routine care given during standard clinical practice, the dosage of anaesthetic drugs is calculated to limit any side effect (nausea and vomiting) that may cause women to be hospitalised on the day of the surgical intervention, all outcomes measured are very relevant for women in general and participants with adverse outcomes (eg, dyspareunia and sexual dysfunction) will be recalled after the end of the study for counselling and therapy. The results of the NOTABLE trial will therefore have a limited generalisability, and their interpretation will be done cautiously. The testing of the safety and the cost-effectiveness will be needed in the longer term using pragmatic multicentre randomised controlled trials or a prospective register.

As suggested by the IDEAL collaboration, more research (large multicentre trials performed by adequately trained surgeons in centres of clinical excellence and large prospective registries cumulating data on the safety of the new technique over many years) and adequate surgical training will be needed before vNOTES can be offered as a standard daily care surgical practice by a majority of gynaecological surgeons for all women bound to undergo removal of one or both adnexa for benign gynaecological disease.

\section{ETHICS AND DISSEMINATION}

The NOTABLE trial will be conducted in accordance with the ethical principles outlined in the latest version of the 'Declaration of Helsinki', the 'Guideline for Good Clinical Practice' and the Belgian Law of 7 May 2004 related to experiments on humans.

All eligible women wishing to participate in the study will receive a detailed patient information document about the study protocol, the aims of the research and the possible adverse events related to the surgical techniques. We will request written informed consent from all participants before randomisation. The principal investigator (JFB) and the coordinating investigator (JJAB) will obtain these consents during a study intake. An adapted informed consent form (online supplementary appendix 1) was drafted based on the template proposed by the FAMHP for clinical research in Belgium. ${ }^{28}$

The protocol of the NOTABLE trial is registered in ClinicalTrials.gov of the US National Institutes of Health as NCT02630329 (online supplementary appendix 2). The study protocol (online supplementary appendix 3) and the informed consent documents have been approved by the Ethics Committee of the Imelda Hospital, Bonheiden, Belgium. The written approval with the Belgian unique study identifier B689201526268 was sent to the FAMHP 
in Brussels. All substantial protocol modifications will be communicated to all trial participants, the hospital's Ethics Committee, ClinicalTrials.gov and the FAMHP.

The NOTABLE trial is a non-commercial and investigator-driven study. The investigators have taken out an insurance policy for medicolegal responsibility related with the conduct of the study from 1 December 2015 to 30 May 2018 in accordance with Article 29 of the Belgian Law of 7 May 2014 related to experiments on humans.

The clinical research forms and all other study-related documents will be stored securely at the study site in locked file cabinets in an area with limited access. All records that contain names or other personal identifiers will be stored separately from study records identified by a code number. Data collection, storage and dissemination will be in accordance with the Belgian Law of 8 December 1992 on the protection of privacy in relation to the processing of personal data and by the Law of 22 August 2002 on patient rights.

At the end of the NOTABLE trial, the complete final data set will be accessible to all trial investigators (the nine authors of the study protocol).

Offering the surgical intervention identified as being most effective or most advantageous after the final analysis of the study data to those women who were allocated to the least effective technique is by nature of the surgical intervention not always possible except for women who had a unilateral surgical intervention. As part of good clinical practice, we will offer post-trial care to women with identified adverse events.

The investigators declare that they have no conflict of interest with respect to the present research.

The NOTABLE trial results will in all circumstances be disseminated through scientific journals and at scientific conference presentations regardless of any positive or negative outcome in relation with the predefined study hypothesis is refuted by the data. All trial investigators will contribute to authorship, following the International Committee of Medical Journal Editors's authorship eligibility guidelines.

\footnotetext{
Author affiliations

${ }^{1}$ Department of Gynaecology, Imelda Hospital, Bonheiden, Belgium

${ }^{2}$ Department of Anaesthesiology, Imelda Hospital, Bonheiden, Belgium

${ }^{3}$ Department of Clinical and Experimental Medicine, KU Leuven-University of Leuven, Leuven, Belgium

${ }^{4}$ Division of Clinical and Experimental Endocrinology, Department of Chronic Diseases, Metabolism and Ageing, KU Leuven-University of Leuven, Leuven, Belgium

${ }^{5}$ Leuven Biostatistics and Statistical Bioinformatics Centre (L-BioStat), KU LeuvenUniversity of Leuven, Leuven, Belgium

${ }^{6}$ Department of Neurosciences, Interfaculty Institute for Family and Sexuality

Studies, KU Leuven-University of Leuven, Leuven, Belgium

${ }^{7}$ Universitaire Vrouwenkliniek, Ghent University Hospital, Ghent, Belgium

${ }^{8}$ The Robinson Institute, The University of Adelaide, Adelaide, South Australia,

Australia

${ }^{9}$ School of Paediatrics \& Reproductive Health, The University of Adelaide, Adelaide, South Australia, Australia

${ }^{10} \mathrm{CEBAM}$, The Centre for Evidence-based Medicine, Cochrane Belgium, KU LeuvenUniversity of Leuven, Leuven, Belgium
}

${ }^{12}$ Academic Centre for General Practice, KU Leuven-University of Leuven, Leuven, Belgium

Acknowledgements We thank the vNOTES investigators' team for taking care of the study participants. We also thank Amanda McPhail for language correction and editing of the manuscript.

Contributors JFB is the surgeon responsible for all interventions in all study participants. JJAB is the outcome assessor. JFB and JJAB conceived the study. PADM and ILR are responsible for the draft of the pain protocol and the anaesthesia for all trial participants. JJAB, JFB, PADM and ILR will be responsible for data collection, quality analysis and storage. PE provided expertise for the sexuality research involved in this clinical trial design. SW provided external review as a content expert. CM provided external peer review on the scientific conduct of the study. AL is responsible for the biostatistics involved in the design and conduct of the trial, reviewed the SAP (Statistical Analysis Plan) of both HALON and NOTABLE and will perform all data analysis for both studies without any involvement of the surgeon (JFB) and the outcome assessor (JJAB). BWJM provided external peer review as a methodology expert. All authors contributed to the refinement of the study protocol and approved the final manuscript. For the economic analyses, we will seek assistance from a Health Economist at the University of Ghent or at the Belgian Health Care Knowledge Centre.

Competing interests None declared.

Patient consent Obtained.

Ethics approval Ethics Committee of Imelda Hospital Bonheiden, protocol no B689201526268, 01/12/2015.

Provenance and peer review Not commissioned; externally peer reviewed.

Data sharing statement The study protocol can be found at ClinicalTrials.gov study reference: NCT02630329.

Open Access This is an Open Access article distributed in accordance with the Creative Commons Attribution Non Commercial (CC BY-NC 4.0) license, which permits others to distribute, remix, adapt, build upon this work non-commercially, and license their derivative works on different terms, provided the original work is properly cited and the use is non-commercial. See: http://creativecommons.org/ licenses/by-nc/4.0/

(c) Article author(s) (or their employer(s) unless otherwise stated in the text of the article) 2018. All rights reserved. No commercial use is permitted unless otherwise expressly granted.

\section{REFERENCES}

1. Kalloo AN, Singh VK, Jagannath SB, et al. Flexible transgastric peritoneoscopy: a novel approach to diagnostic and therapeutic interventions in the peritoneal cavity. Gastrointest Endosc 2004;60:114-7.

2. Rao GV, Reddy DN, Banerjee R. NOTES: human experience. Gastrointest Endosc Clin N Am 2008;18:361-70.

3. Rolanda C, Lima E, Pêgo JM, et al. Third-generation cholecystectomy by natural orifices: transgastric and transvesical combined approach (with video). Gastrointest Endosc 2007;65:111-7.

4. Rattner D, Kalloo A. ASGE/SAGES Working Group. ASGE/SAGES working group on natural orifice translumenal endoscopic surgery. October 2005. Surg Endosc 2006;20:329-33.

5. Santos BF, Hungness ES. Natural orifice translumenal endoscopic surgery: progress in humans since white paper. World $J$ Gastroenterol 2011;17:1655-65.

6. Tolcher MC, Kalogera E, Hopkins MR, et al. Safety of culdotomy as a surgical approach: implications for natural orifice transluminal endoscopic surgery. JSLS 2012;16:413-20.

7. Uccella S, Cromi A, Bogani G, et al. Transvaginal specimen extraction at laparoscopy without concomitant hysterectomy: our experience and systematic review of the literature. J Minim Invasive Gynecol 2013;20:583-90.

8. Lee C-L, Wu K-Y, Su H, et al. Transvaginal Natural-Orifice Transluminal Endoscopic Surgery (NOTES) in adnexal procedures. $J$ Minim Invasive Gynecol 2012;19:509-13.

9. Reynders A, Baekelandt J. Adnexectomy by poor man's transvaginal NOTES. Gynecol Surg 2015;12:207-11.

10. Van Peer S, Baekelandt J. Natural orifice transluminal endoscopic surgery (NOTES) salpingectomy for ectopic pregnancy: a first series 
demonstrating how a new surgical technique can be applied in a low-resource setting. Gynecol Surg 2015;12:299-302.

11. Baekelandt J. Total vaginal NOTES hysterectomy: a new approach to hysterectomy. J Minim Invasive Gynecol 2015;22:1088-94.

12. Baekelandt J, De Mulder PA, Le Roy I, et al. HALON-hysterectomy by transabdominal laparoscopy or natural orifice transluminal endoscopic surgery: a randomised controlled trial (study protocol). BMJ Open 2016;6:e011546.

13. Shamseer L, Moher D, Clarke M, et al. Preferred reporting items for systematic review and meta-analysis protocols (PRISMA-P) 2015: elaboration and explanation. BMJ 2015;349:97647.

14. National Institute for Health Research. PROSPERO International prospective register of systematic reviews. http://www.crd.york.ac. uk/prospero/

15. Barkun JS, Aronson JK, Feldman LS, et al. Evaluation and stages of surgical innovations. Lancet 2009;374:1089-96.

16. Ergina PL, Cook JA, Blazeby JM, et al. Challenges in evaluating surgical innovation. Lancet 2009;374:1097-104.

17. McCulloch P, Altman DG, Campbell WB, et al. No surgical innovation without evaluation: the IDEAL recommendations. Lancet 2009;374:1105-12.

18. Gargon E, Gurung B, Medley N, et al. Choosing important health outcomes for comparative effectiveness research: a systematic review. PLoS One 2014;9:e99111.

19. http://www.comet-initiative.org/studies/searchresults
20. Todd KH, Funk KG, Funk JP, et al. Clinical significance of reported changes in pain severity. Ann Emerg Med 1996;27:485-9.

21. Dindo D, Demartines N, Clavien PA. Classification of surgical complications: a new proposal with evaluation in a cohort of 6336 patients and results of a survey. Ann Surg 2004;240:205-13.

22. Aerts L, Enzlin P, Verhaeghe J, et al. Psychologic, relational, and sexual functioning in women after surgical treatment of vulvar malignancy: a prospective controlled study. Int J Gynecol Cancer 2014;24:372-80.

23. Moseley JB, O'Malley K, Petersen NJ, et al. A controlled trial of arthroscopic surgery for osteoarthritis of the knee. N Engl J Med 2002;347:81-8.

24. Freedman B. Equipoise and the ethics of clinical research. $N$ Engl $J$ Med 1987;317:141-5.

25. Wang PH, Lee WL, Juang CM, et al. Excision of mature teratoma using culdotomy, with and without laparoscopy: a prospective randomised trial. BJOG 2001;108:91-4.

26. Schmitt A, Crochet P, Knight S, et al. Single port laparoscopy versus conventional laparoscopy in benign adnexal diseases: a systematic review and meta-analysis. J Minim Invasive Gynecol 2017;1553:4650.

27. Schulz KF, Altman DG, Moher D. CONSORT 2010 Statement: updated guidelines for reporting parallel group randomized trials. Ann Intern Med 2010;152:726-8.

28. AFMPS. Take a medicine if you want to have a baby, are pregnant or breastfeeding? www.afmps.be 